\title{
SKINCARE AND QUALITY OF LIFE AMONG OLDER RESIDENTS LIVING IN JAPANESE NURSING HOMES
}

\author{
M. NAGAE 1 , T. MITSUTAKE 2 , M. SAKAMOTO

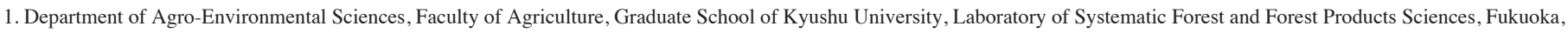 \\ Japan; 2. Department of Physical Therapy, Fukuoka International University of Health and Welfare, Fukuoka, Japan; 3. Division of Medical Education Development, Research \& \\ Education Center for Community Medicine, Faculty of Medicine, Saga University, Saga, Japan. Corresponding author: Masumi Nagae,: Department of Agro-Environmental Sciences, \\ Faculty of Agriculture, Graduate School of Kyushu University 744, Motooka, Nishi-ku, Fukuoka 819-0395, Japan, Phone/Fax: +81-92-802-4673, nagae.masumi.258@kyushu-u.ac.jp
}

\begin{abstract}
This study aimed to clarify the practices of skincare such as bathing, face-washing, and using of skincare products, among older adults living in nursing homes in Japan. From July to August 2017, questionnaire surveys were mailed to 343 long-term care facilities in one prefecture in Japan, and 105 facilities returned the survey. Management policies on the use of facial skincare products was significantly associated with the number of resident/caregiver ratio. Meanwhile, residents in nursing homes with higher numbers of caregivers washed their faces and bathed at lower rates. There were no correlations between the number of resident/caregiver ratio, face-washing, and bathing times. When older residents could not independently perform their skin cleansing and care, that care is likely to happen less often. Sufficient manpower for the support of older residents' skincare will improve the quality of life in long-term nursing facilities.
\end{abstract}

Key words: Activities of daily living (ADL), nursing home, skin cleansing, skincare, quality of life.

\section{Introduction}

Japan is a country with one of the fastest aging populations. In 2017 , the older population (65 years old or older) was 3.52 million (1).

Quality of life (QOL) among older populations has become an important outcome measure because it can give a more comprehensive "view of health care in the country and the specific growing population" (2).

Skincare in late adulthood has come to be recognized as one of the cornerstone nursing practices (3). However, given the limited number of studies, it is unclear how skincare has been practiced in public health worldwide. Moreover, it is also unclear how the basic ADLs with regard to skincare for older residents in nursing homes are currently being provided. This study aimed to collect practical data in Japanese nursing homes on the basic ADLs regarding skincare such as bathing, face-washing and the use of skincare products, and deduced the surroundings of those practices.

\section{Methods}

\section{Study setting and participants}

A questionnaire survey was conducted at 343 long-term care facilities that were registered in a prefecture in Japan, from July to August 2017. Once facilities agreed to voluntarily participate in this study and provided their consent, respective administrators answered the questionnaires. We referred to the research protocol from a previous study which had carried out in Germany (4). Meanwhile, we referred to the response rates from another previous study regarding Japanese nursing homes, which reported that those rates of domestic surveys were usually around $30 \%$ (5). The study protocol was in keeping with the ethical standards laid down in the Declaration of Helsinki and was approved by the Faculty of Medicine, Saga University Sciences Committee on Ethics (29-26, 2017). All participants provided informed consent before participating in the study.

\section{Variables}

To gain an overview of the facilities, data regarding the type of facility and the number of employees working in each job type at the facilities were also collected.

The variables of residents' skin cleansing and care were assessed from questions which were re-formulated based on a previous study (6). We inquired about how often the residents washed their face per day and bathed per week, with or without help. We also asked about the facilities' policies regarding their use of skin cleansing and application products on residents' faces and bodies (See appendix for more information).

\section{Statistical analyses}

First, types and numbers of facilities in this sample were compared descriptively with those from the prefecture and Japan (7-9). Second, the basic information on nursing homes that were surveyed in this study descriptively. In the present study, 94-96\% of facilities provided older residents with cleansing-products of hair and skin on their own responsibility. On the other hand, skincare products were provided according to each facility's management policy. Therefore, we categorized the facilities based on the use of face-application products. Facilities where residents individually provided their own face-application products or they did not use any product were defined as Individual management; facilities where a face- 
Table 1

Nursing home characteristics

\begin{tabular}{|c|c|c|c|c|c|c|c|c|}
\hline & \multirow{3}{*}{$\begin{array}{l}\text { *All nursing homes } \\
\text { n(\%) }\end{array}$} & \multicolumn{7}{|c|}{ Study nursing homes } \\
\hline & & \multicolumn{2}{|r|}{ Total } & \multicolumn{2}{|c|}{$\begin{array}{c}\text { §Individual } \\
\text { skincare management }\end{array}$} & \multicolumn{2}{|c|}{$\begin{array}{c}\text { §Nursing home } \\
\text { skincare management }\end{array}$} & \multirow[b]{2}{*}{$\dagger \mathbf{P}$ value } \\
\hline & & $\mathbf{n}(\%)$ & Median $(0.25-0.75)$ & $\mathbf{n}(\%)$ & Median (0.25-0.75) & $\mathbf{n}(\%)$ & Median (0.25-0.75) & \\
\hline Number & $343(100)$ & $105(100)$ & & $81(100)$ & & $20(100)$ & & \\
\hline \multicolumn{9}{|l|}{ §Type } \\
\hline Nursing home & $224(65.3)$ & $55(53.4)$ & & $42(51.9)$ & & $11(55.0)$ & & \\
\hline Special Nursing home & $57(16.6)$ & $27(26.2)$ & & 21(25.9) & & $6(30.0)$ & & \\
\hline Long-Term Care Health Facility & $41(12.0)$ & 14(13.6) & & 12(14.8) & & $2(10.0)$ & & \\
\hline Medical Long-Term Care Facility & $21(6.1)$ & $7(6.8)$ & & $6(7.4)$ & & $1(5.0)$ & & \\
\hline Number of older residents & & & $42.0(17.0-59.0)$ & & $42.5(17.8-64.3)$ & & $37.0(13.0-54.5)$ & 0.389 \\
\hline Number of caregivers & & & $15.0(7.0-27.0)$ & & $15.0(6.8-27.0)$ & & $11.0(7.5-27.5)$ & 0.838 \\
\hline Number of staff except for caregivers & & & $8.0(4.0-12.0)$ & & $8.0(4.0-12.0)$ & & $7.0(4.0-11.0)$ & 0.664 \\
\hline $\begin{array}{l}\text { Number of taking care of older } \\
\text { residents/one caregiver }\end{array}$ & & & $2.1(1.7-3.0)$ & & $2.2(1.8-3.1)$ & & $1.8(1.4-2.2)$ & 0.031 \\
\hline $\begin{array}{l}\text { Face washing times/day of older } \\
\text { residents with need for help }\end{array}$ & & & $1.0(1.0-2.0)$ & & $1.0(1.0-2.0)$ & & $1.0(1.0-1.8)$ & 0.231 \\
\hline $\begin{array}{l}\text { Face washing times/day of older } \\
\text { residents without need for help }\end{array}$ & & & $1.0(1.0-2.0)$ & & $1.0(1.0-1.9)$ & & $1.0(1.0-2.0)$ & 0.703 \\
\hline $\begin{array}{l}\text { Bath times/week of older residents } \\
\text { with need for help }\end{array}$ & & & $2.5(2.0-3.0)$ & & $2.5(2.0-3.0)$ & & $2.8(2.0-3.0)$ & 0.501 \\
\hline $\begin{array}{l}\text { Bath times/week of older residents } \\
\text { without need for help }\end{array}$ & & & $2.5(2.0-3.0)$ & & $2.5(2.0-3.0)$ & & $3.0(2.0-4.1)$ & 0.310 \\
\hline
\end{tabular}

application product was provided for the residents were defined as Nursing home management. Third, the Mann-Whitney U-test was performed to compare basic characteristics of nursing homes and characteristics of nursing homes' skincare, by two categories based on the use of face-application products. Finally, Spearman's rank correlation analysis was performed between number of caregivers, number of resident/caregiver ratio, and each item of characteristics of nursing homes' skincare to analyse each association. A p-value of less than 0.05 was considered as a statistically significant difference. The analyses were performed using SPSS 25.0 J for Windows (SPSS, Chicago).

\section{Results}

\section{Sample}

Of the 343 long-term care facilities contacted, 105 facilities $(31 \%)$ agreed to participate in this study and provided informed consent. Table 1 shows the number of nursing homes in a prefecture in Japan, and those which took part in the present study, and basic information and characteristics of nursing homes' skincare in this sample and by two categories based on types of management of face-application products usage.

The proportions of different types of facilities, such as nursing home, special nursing home, long-term care health facility, and medical long-term care facility were as follows: the sample survey $2017(53.4 \%, 26.2 \%, 13.6 \%, 6.8 \%$, respectively), Statistics A Prefecture 2017 (65.3\%, $16.6 \%$, $12.0 \%, 6.1 \%$, respectively) (Table 1), and Japanese national statistics from 2017 (50.2\%, 29.3\%, 16.1\%, 4.4\%, respectively).

Analyses between individual-management-type and nursing-home-management-type facilities regarding the use of face-application products and manpower

There was no significant difference between characteristics of nursing homes' skincare by two categories based on the use of face-application products (Table 1). However, there was a significantly higher older residents/caregiver ratio in individualmanagement facilities [2.2 (1.8-3.1)] compared to nursinghome-management facilities [1.8 (1.4-2.2)] $(\mathrm{p}=0.031)$.

Relationship between face-washing and bathing frequency and manpower environment in facilities

Table 2 shows the result of correlations between number of caregivers, number of older residents/caregiver ratio, and each item of the characteristics of nursing homes' skincare. There was a significant negative correlation between number of caregivers and the number of older residents/caregiver ratio (correlation coefficient $[R]=-0.319, p=0.002$ ). Number of caregivers had a significant negative correlation with both 
THE JOURNAL OF NURSING HOME RESEARCH SCIENCESC

Table 2

Spearman's rank correlation coefficients between manpower at facilities and face-washing and bathing frequencies

\begin{tabular}{|c|c|c|c|c|c|c|}
\hline & & 1 & 2 & 3 & 4 & 5 \\
\hline 1 & Number of caregivers & & & & & \\
\hline 2 & Number of taking care of older residents/one caregiver & $-0.319 *$ & & & & \\
\hline 3 & Older residents with need for help face washing times/day & $-0.283^{*}$ & 0.003 & & & \\
\hline 4 & Older residents without need for help face washing times/day & $-0.230 *$ & 0.124 & $0.419 *$ & & \\
\hline 5 & Older residents with need for help bath times/week & $-0.461 *$ & -0.043 & $0.308 *$ & 0.052 & \\
\hline 6 & Older residents without need for help bath times/week & $-0.485^{*}$ & 0.031 & $0.430 *$ & 0.153 & $0.891 *$ \\
\hline
\end{tabular}

residents with need for help $(\mathrm{R}=-0.283, \mathrm{p}=0.005)$ and without need for help ( $\mathrm{R}=-0.230, \mathrm{p}=0.042)$ to perform daily facewashing. Similarly, number of caregivers had a significant negative correlation with both residents with need for help $(\mathrm{R}$ $=-0.461, \mathrm{p}<0.001)$ and without need for help $(\mathrm{R}=-0.485$, $\mathrm{p}<0.001)$ to take their weekly bath.

Contrastingly, there was no significant correlation between number of resident/caregiver ratio and daily face-washing frequency for both residents with need for help $(R=0.003$, $\mathrm{p}=0.980)$ and without need for help $(\mathrm{R}=0.124, \mathrm{p}=0.294)$. Similarly, there was no significant correlation between number of resident/caregiver ratio and weekly bath frequency for both residents who needed for help $(-0.043, \mathrm{p}=0.685)$ or those who did not need for help $(\mathrm{R}=0.031, \mathrm{p}=0.790)$.

\section{Discussion}

Residents in facilities bathed and washed their faces fewer times than other people performed those activities at their homes (10). Japan is aging ever faster, and understaffing of welfare work has been a serious problem (11). However, no significant correlation was found between the resident/ caregiver ratio and the frequency of face-washing or bathing. Thus, lack of manpower may not be a direct factor related to the limited number of opportunities for face-washing or bathing. In contrast, there was a significant difference of the number of taking care of older residents between two categories based on types of management of face-application products usage.

Since the late 1990s, nursing care in Japan has been developed in terms of individual and unit care, which has created a homelike environment (12). However, the government recommendation for bathing merely states, "at least twice a week using an appropriate method", and there are no guidelines for the quality of bathing.

Even if facilities observe minimum standard rules, the quality of bathing practices differs from that in regular homes. Most facilities tend to have less frequency of daily face-washing and weekly bath. Their face-washing or bathing policies might have been developed to observe the guidelines on the minimum number of bathing times (13), even if there are enough staff members to support older residents. In contrast, management policy on the use of facial skincare products was significantly associated with the number of the resident/ caregiver ratio. Individual-management-type facilities showed a 2.2 resident/caregiver ratio, on the other hand, Nursing-homemanagement-type facilities showed 1.8 resident/caregiver ratio. This small difference affected residents' opportunities to use skincare products. Enhancing QOL is an important part of older adults' healthcare (3), and it has gained more attention in recent years (8). Moreover, policies around older residents' care have been designed to support personal dignity (14). Additionally, it has reported that maintaining healthy skin was associated with better mental and emotional wellbeing (15). However, if facilities always leave older adults to their own skincare without looking at the personal environment, the policy is not consistent with supporting personal dignity and wellbeing.

\section{Limitations}

This study has a few limitations. First, the present study was conducted at long-term care facilities in only one prefecture in Japan, and the facilities participated voluntarily. We cannot completely remove the possibility of selection bias. Secondly, this study was a cross-sectional survey that focused on the lifestyles of nursing home residents, so these results should be cautiously applied to clinical practice.

\section{Conclusion}

Regardless of these limitations, to the best of our knowledge, this was the first study to collect practical data on skin cleansing and care in Japanese nursing homes. As for the support for activities of daily living in long term care, facilities do not only obey guidelines, but also should examine how best to support residents from the viewpoint of personal dignity and the individual care.

Study design: MN, TM, MS; data collection and analysis: MN and manuscript preparation: $\mathrm{MN}, \mathrm{MS}$.

Conflict of Interest: The authors have no potential conflicts of interest to disclose. 


\section{SKINCARE AND QUALITY OF LIFE IN NURSING HOMES}

Funding: This work was supported by JSPS KAKENHI under Grant number JP17K09218.

Ethical standards: The study protocol was approved by the Faculty of Medecine, Saga University Sciences Committee on Ethics (29-26, 2017).

\section{References}

1. 2018 edition aging society white paper (whole version). Aging and future. https:// www8.cao.go.jp/kourei/whitepaper/w-2018/html/gaiyou/s1_1.html. Accessed 30 November 2018

2. Netuveli, G., \& Blane, D. Quality of life in older ages. British Medical Bulletin. 2008; 85 (1): 113-126. doi: 10.1093/bmb/ldn003

3. Cowdell, F., \& Steventon, K. Skin cleansing practices for older people: a systematic review. International Journal of Older People Nursing 2015; 10 (1): 3-13. doi: 10.1111/opn.12041

4. Kottner, J., Dassen, T., \& Lahmann, N. A. Pressure ulcers in German nursing homes: frequencies, grades, and origins. Zeitschrift fur Gerontologie und Geiatrie, 2011; 44 (5): 318-322. doi: 10.1007/s00391-011-0179-0

5. Sawamura K., Nakashima T., Nakanishi M. Provision of individualized care and built environment of nursing homes in Japan. Archives of Gerontology and Geriatrics. 2013; 56 (3)3: 416-424. doi.org/10.1016/j.archger.2012.11.0096. Kottner, J., Lichterfeld, A., \& Blume-Peytavi, U. Maintaining skin integrity in the aged: systematic review. British Journal of Dermatology 2013; 169 (3): 528-542. doi: 10.1111/bjd.12469

7. Ministry of Health, Labor and Welfare 2017a https://www.mhlw.go.jp/topics/kaigo/ osirase/jigyo/m17/1708.html. Accessed 30 November 2018

8. Statistics of Japan 2017 https://www.e-stat.go.jp/dbview?sid=0003215842. Accessed 30 November 2018

9. Welfare and Medical Service Agency 2017. http://www.wam.go.jp/content/wamnet/ pcpub/kourei/service/. Accessed 31 May 2017

10. Morishita, M., Kawasaki, R., Nakao, R., \& Hanzawa, S. The correlation of the QOL of the elderly women aged 75 years and older living alone with their length of residence, their lifestyles and their health. Hokengakukenkyu 2007; 19 (2): 31-41(in Japanese). http://hdl.handle.net/10069/9785

11. Welfare and Medical Service Agency 2017b, https://www.wam.go.jp/hp/wp-content/ uploads/H3009.pdf. Accessed 30 November 2018

12. Ministry of Health, Labor and Welfare 2017b https://www.mhlw.go.jp/toukei/saikin/ hw/kaigo/service17/dl/kekka-gaiyou.pdf. Accessed 30 November 2018

13. A welfare for aged-related laws and ordinances meeting for the study 2013 Criteria about the staff of the designated care facility for social welfare for aged, facilities and the administration. Welfare for aged-related collection of notices of laws and ordinances 2013 edition, Tokyo, pp795-796.

14. Coventry, M. L. Care with dignity: A concept analysis journal of gerontological nursing. Thorofare 2006; 32 (5): 42-48. https://doi.org/10.3928/00989134-2006050108

15. Blume-Peytavi, U., Kottner, J., Sterry, W. et al. Age-associated skin conditions and diseases: current perspectives and future options. Gerontologist 2016; 56 (2): 230-242. doi: 10.1093/geront/gnw003 